\title{
MIMO-Assisted Hard Versus Soft Decoding-and-Forwarding for Network Coding Aided Relaying Systems
}

\author{
Kyungchun Lee and Lajos Hanzo, Fellow, IEEE
}

\begin{abstract}
This paper proposes two types of new decoding algorithms for a network coding aided relaying (NCR) system, which adopts multiple antennas at both the transmitter and receiver. In the NCR system, the relay station (RS) decodes the data received from both the base station (BS) as well as from the mobile station (MS) and combines the decoded signals into a single data stream before forwarding it to both. In this paper, we consider the realistic scenario of encountering decoding errors at the RS, which results in erroneous forwarded data. Under this assumption, we derive decoding algorithms for both the BS and the MS in order to reduce the deleterious effects of imperfect decoding at the RS. We first propose a decoding algorithm for a hard decision based forwarding (HDF) system. Then, for the sake of achieving further performance improvements, we also employ soft decision forwarding (SDF) and propose a novel error model, which divides the error pattern into two components: hard and soft errors. Given this error model, we then modify the HDF decoder for employment in SDF systems. We also derive estimation algorithms for their parameters that are required for the efficient operation of the proposed decoders. Our simulation results show that the proposed algorithms provide substantial performance improvements in terms of the attainable packet error rate as a benefit of our more accurate error model.
\end{abstract}

Index Terms-Network coding, relaying, cooperative communication, multiple-input multiple-output (MIMO) system.

\section{INTRODUCTION}

$\mathbf{M}$ ULTIPLE-INPUT multiple-output (MIMO) diversity techniques are capable of achieving substantial diversity gains, provided that their elements experience independent or moderately correlated fading. However, in practical communication systems, multiple antennas are typically allocated insufficiently far apart, especially at the mobile station (MS) but often even at the base station (BS), which results in correlated spatial channels. Hence the spatial diversity promise of independently faded signals is often eroded. In order to cope with this problem, MIMO systems can be combined with adaptive modulation [1] as well as cooperative and relay station (RS) aided transmission schemes [2]-[6]. In relaying

Manuscript received January 13, 2008; revised May 5, 2008; accepted July 8, 2008. The associate editor coordinating the review of this paper and approving it for publication was A. Grant.

K. Lee is with Samsung Electronics, P.O. Box 105, Suwon, Korea (e-mail: kclee@sclab.kaist.ac.kr).

L. Hanzo is with the School of Electronics and Computer Science, University of Southampton, SO17 1BJ Southampton, U.K. (e-mail: lh@ecs.soton.ac.uk).

This work was supported by the Korea Research Foundation Grant funded by the Korean Government (MOEHRD) (KRF-2007-357-D00110) and by the EU under the auspices of the OPTIMIX project.

Digital Object Identifier 10.1109/T-WC.2009.080048

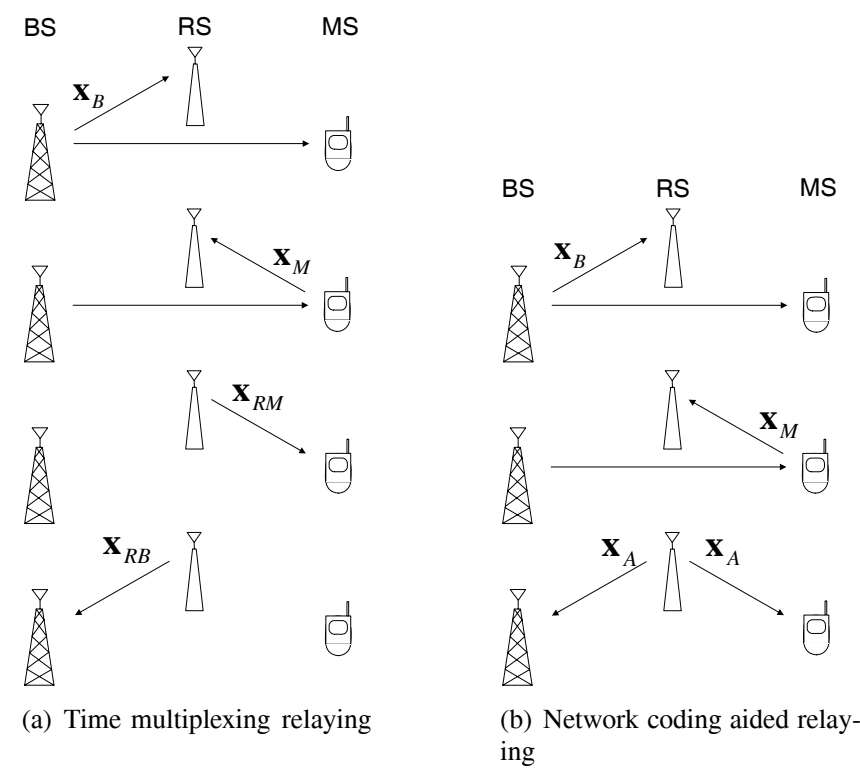

Fig. 1. Relay system model

systems a MS receives both the two-hop downlink (DL) signal via the RS as well as the directly detected signal of the BS. Since these two signals typically arrive via completely different paths, the correlation between the fading of these channels is typically low. Furthermore, the RS is capable of extending the cell area and/or improving the quality of the reception at the cell-edge, which results in requiring a reduced number of BSs for maintaining seamless coverage.

Naturally, the relaying of the DL signal requires additional resources. For example, as shown in Fig. 1(a), the traditional time multiplexing based relaying scheme [5] requires four time slots (TSs) for duplex communications, which is twice higher than that of the direct link between a MS and a BS. Therefore, relaying schemes requiring lower resources have been investigated in [7]-[11]. The network coding aided relaying (NCR) scheme of Fig. 1(b) jointly encodes the signals received from two source nodes (such as a MS and a BS) into a single coded stream $\mathrm{x}_{A}$ [7], [8]. Once the RS received the data to be conveyed to the BS and MS in the uplink (UL) and DL in its two receiver TSs, it forwards their jointly encoded data to both the MS and the BS in the same time slot, which is seen as a DL slot for the MS and a UL slot for the BS, rather than independently transmitting their respective data in a different TS. Accordingly, the NCR system requires only three TSs, 


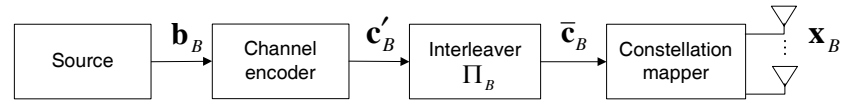

(a) DL transmitter at the BS

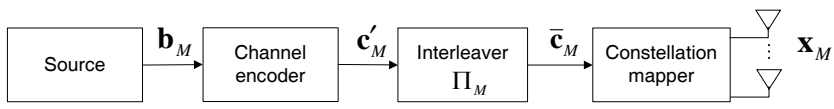

(b) UL transmitter at the MS

Fig. 2. Source nodes of the NCR system

which leads to a $33 \%$ throughput enhancement compared to the traditional relaying scheme of Fig. 1(a). Naturally, this TS reduction is achieved at the cost of potential error-propagation, which is reminiscent of the that experienced in differentially encoded systems.

The NCR is based on the decode-and-forward (DF) relaying strategy [4], [12]. In DF relaying, the relay node fully decodes the received signal and forwards its re-encoded version in order to avoid the noise amplification of amplify-and-forward (AF) schemes. However, upon slicing the transmitted signal, the DF relay discards the soft information, which would be helpful at the destination node. To take the advantage of DF relaying, while also retaining the soft information at the same time, soft decision aided forwarding (SDF) techniques have been proposed in [13], [14]. In SDF relaying, the relay retransmits soft-valued estimates of the decoded signals, rather than their hard-decision based sliced versions in order to retain the soft information.

In this paper, we propose novel decoding algorithms for the destination nodes of NCR systems employing multiple antennas. In Section III-A, we derive an optimal decoding algorithm for hard decision forwarding (HDF) aided MIMO relaying systems, which transmits hard-decision values from the RS. Then, in Section III-B we extend this algorithm to the SDF aided MIMO relaying. When deriving the proposed algorithms, we employ an accurate error model for the sake of attaining a valuable performance improvement.

This paper is organized as follows. Section II describes the NCR system model, while Section III derives the proposed decoding algorithms. In Section IV our simulation results are provided for performance comparisons. Finally, we present our conclusions in Section V.

\section{SYSTEM MODEL}

We assume that the BS, the MS, and the RS have the same number of transmit/receive antennas for notational convenience. Furthermore, we assume that the number of channel uses is fixed in both the UL and DL ${ }^{1}$. As shown in Fig. 2, we consider vertically encoded MIMO systems [15], [16], where the BS encodes the DL information bit stream $\mathbf{b}_{B}$ into a codeword $\mathbf{c}_{B}^{\prime}$, which is then interleaved to obtain $\overline{\mathbf{c}}_{B}=\left\{\overline{\mathbf{c}}_{B}^{1}, \overline{\mathbf{c}}_{B}^{2}, \cdots, \overline{\mathbf{c}}_{B}^{l}, \cdots, \overline{\mathbf{c}}_{B}^{T}\right\}$, where $T$ is the number of channel uses and $\overline{\mathbf{c}}_{B}^{l}=\left\{\bar{c}_{B, 1}, \bar{c}_{B, 2}, \cdots, \bar{c}_{B, N_{t} M_{c}}\right\}$ denotes the $N_{t} \cdot M_{c}$ coded bits to be transmitted during the $l$ th channel

\footnotetext{
${ }^{1}$ In realistic environments where the packet sizes are different in the UL and DL, we can employ zero-padding or repetition coding to make them equal-length when joint encoding of the UL and DL signals is performed at the RS [7].
}

use. Here, $N_{t}$ denotes the number of transmit antennas and $M_{c}$ is the number of coded bits associated with a single modulated symbol. Then, $\overline{\mathbf{c}}_{B}^{l}$ is mapped to the $N_{t}$-element MIMO symbol $\mathbf{x}_{B} \in \mathcal{C}^{N_{t}}$, which is transmitted across $N_{t}$ transmit antennas.

During the $l$ th channel use of the first TS, the signals encountered at the RS and the MS are formulated as ${ }^{2}$

$$
\begin{aligned}
\mathbf{y}_{B R} & =\mathbf{H}_{B R} \mathbf{x}_{B}+\mathbf{v}_{B R}, \\
\mathbf{y}_{B M} & =\mathbf{H}_{B M} \mathbf{x}_{B}+\mathbf{v}_{B M},
\end{aligned}
$$

respectively, where $\mathbf{y}_{B R}$ and $\mathbf{y}_{B M}$ denote $\left(N_{r} \times 1\right)$ dimensional complex-valued received signal vectors at the RS and the MS, while $\mathbf{H}_{B R}$ and $\mathbf{H}_{B M}$ represent the $\left(N_{r} \times N_{t}\right)$ element complex-valued channel matrices, where $N_{r}$ indicates the number of receive antennas. Furthermore, $\mathbf{v}_{B R}$ and $\mathbf{v}_{B M}$ denote the $\left(N_{r} \times 1\right)$-element noise vectors of independent zeromean complex Gaussian entries having a variance of $\sigma_{v}^{2} / 2$ per dimension, i.e., $\mathbf{v}_{B R}, \mathbf{v}_{B M} \sim \mathcal{C N}\left(\mathbf{0}_{N_{r}}, \sigma_{v}^{2} \mathbf{I}_{N_{r}}\right)$, where $\mathbf{0}_{N_{r}}$ is an $\left(N_{r} \times 1\right)$-element all-zero vector and $\mathbf{I}_{N_{r}}$ is an $\left(N_{r} \times N_{r}\right)$ element identity matrix.

Similarly, as shown in Fig. 2(b), the MS encodes the vector $\mathbf{b}_{M}$ of UL information bits into $\mathbf{c}_{M}^{\prime}$ and interleaves it to obtain $\overline{\mathbf{c}}_{M}=\left\{\overline{\mathbf{c}}_{M}^{1}, \overline{\mathbf{c}}_{M}^{2}, \cdots, \overline{\mathbf{c}}_{M}^{l}, \cdots, \overline{\mathbf{c}}_{M}^{T}\right\}$. Finally, $\overline{\mathbf{c}}_{M}^{l}$ is converted to a symbol vector $\mathbf{x}_{M}$ before commencing transmission. During the $l$ th channel use of the second TS, the signals received at the RS and the BS become

$$
\begin{aligned}
& \mathbf{y}_{M R}=\mathbf{H}_{M R} \mathbf{x}_{M}+\mathbf{v}_{M R}, \\
& \mathbf{y}_{M B}=\mathbf{H}_{M B} \mathbf{x}_{M}+\mathbf{v}_{M B},
\end{aligned}
$$

respectively, where $\mathbf{H}_{M R}$ and $\mathbf{H}_{M B}$ represent the $\left(N_{r} \times N_{t}\right)$ element complex-valued channel matrices. Furthermore, $\mathbf{v}_{M R}$ and $\mathbf{v}_{M B}$ indicate the noise vectors of $\mathbf{v}_{M R}, \mathbf{v}_{M B} \sim$ $\mathcal{C N}\left(\mathbf{0}_{N_{r}}, \sigma_{v}^{2} \mathbf{I}_{N_{r}}\right)$.

As portrayed in Fig. 3, the RS's estimates $\mathbf{c}_{B}^{\prime}$ and $\mathbf{c}_{M}^{\prime}$ are generated by the iterative detection/decoding (IDD) aided MIMO systems [15], [16]. If $\mathbf{c}_{B}^{\prime}$ and $\mathbf{c}_{M}^{\prime}$ are perfectly decoded, then the RS combines $\mathbf{c}_{B}^{\prime}$ and $\mathbf{c}_{M}^{\prime}$ using the elementwise XOR operation into a composite packet ${ }^{3}$ and sends the resultant message to the interleaver $\Pi_{R}(\cdot)$ in order to obtain $\mathbf{c}_{A}=\left\{\mathbf{c}_{A}^{1}, \mathbf{c}_{A}^{2}, \cdots, \mathbf{c}_{A}^{l}, \cdots, \mathbf{c}_{A}^{T}\right\}$, where

$$
\mathbf{c}_{A}^{l}=\left\{c_{A, 1}, c_{A, 2}, \cdots, c_{A, m}, \cdots, c_{A, N_{t} M_{c}}\right\}
$$

represents a bit stream of length $N_{t} \cdot M_{c}$ to be forwarded to the destination nodes during the $l$ th channel use of the third TS.

The MS receives the combined signal of $\mathbf{c}_{A}=\mathbf{c}_{B} \oplus \mathbf{c}_{M}$, where $\mathbf{c}_{B}$ and $\mathbf{c}_{M}$ are defined as $\mathbf{c}_{B}=\Pi_{R}\left(\mathbf{c}_{B}^{\prime}\right)$ and $\mathbf{c}_{M}=$ $\boldsymbol{\Pi}_{R}\left(\mathbf{c}_{M}^{\prime}\right)$, respectively, while $\oplus$ denotes the element-wise XOR operation. To decode $\mathbf{c}_{B}$, we perform the IDD algorithm of Fig. 4. When applying the IDD, we first generate the loglikelihood ratio (LLR) $\mathbf{L}_{R M}^{e, 1}\left(\mathbf{c}_{A}\right)$ for the coded bit vector $\mathbf{c}_{A}$ using the MIMO detector. Before inserting $\mathbf{L}_{R M}^{e, 1}\left(\mathbf{c}_{A}\right)$ into the channel decoder, we remove the effect of $\mathbf{c}_{M}$, which is already

\footnotetext{
${ }^{2}$ For notational convenience, we omit the channel use index throughout the paper, when it does not cause any confusion.

${ }^{3}$ The XOR operation essentially creates the difference of the MS's and BS's transmitted signals, hence the RS's transmission may be viewed as sending to both destinations, namely the MS and the BS, a sequence having a binary one in the transmitted frame, where the sequence destined for their reception is different from their own transmitted sequence.
} 


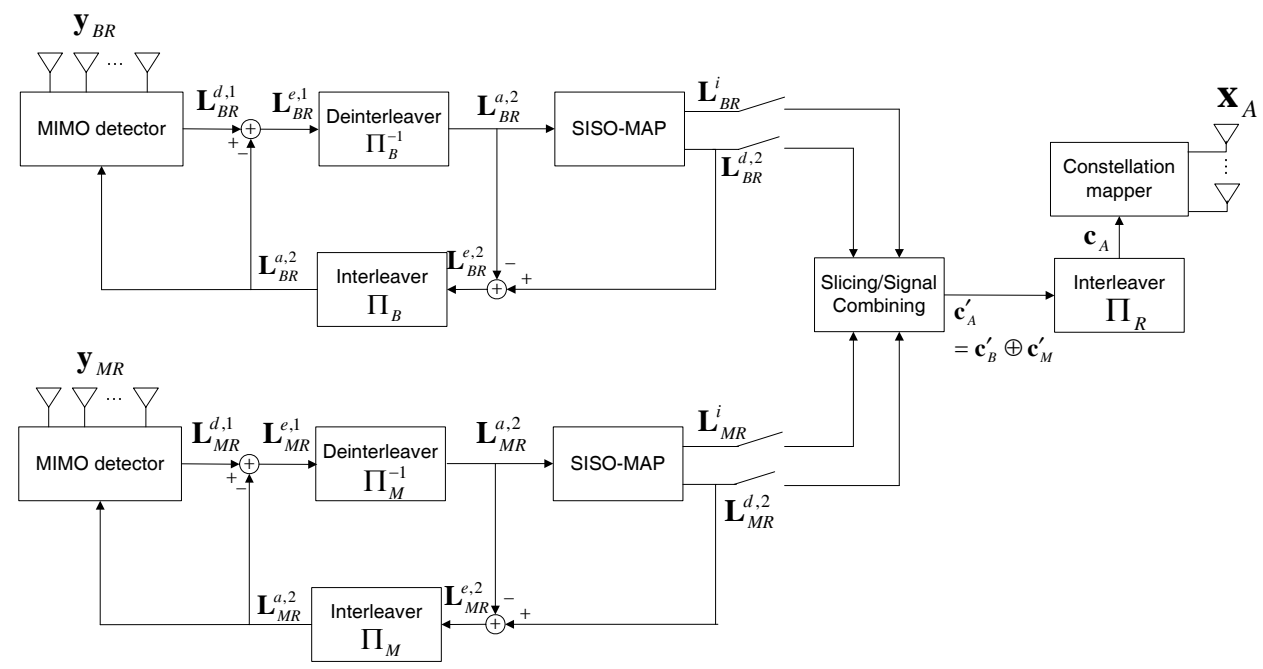

Fig. 3. Block diagram of the receiver at the RS in NCR systems

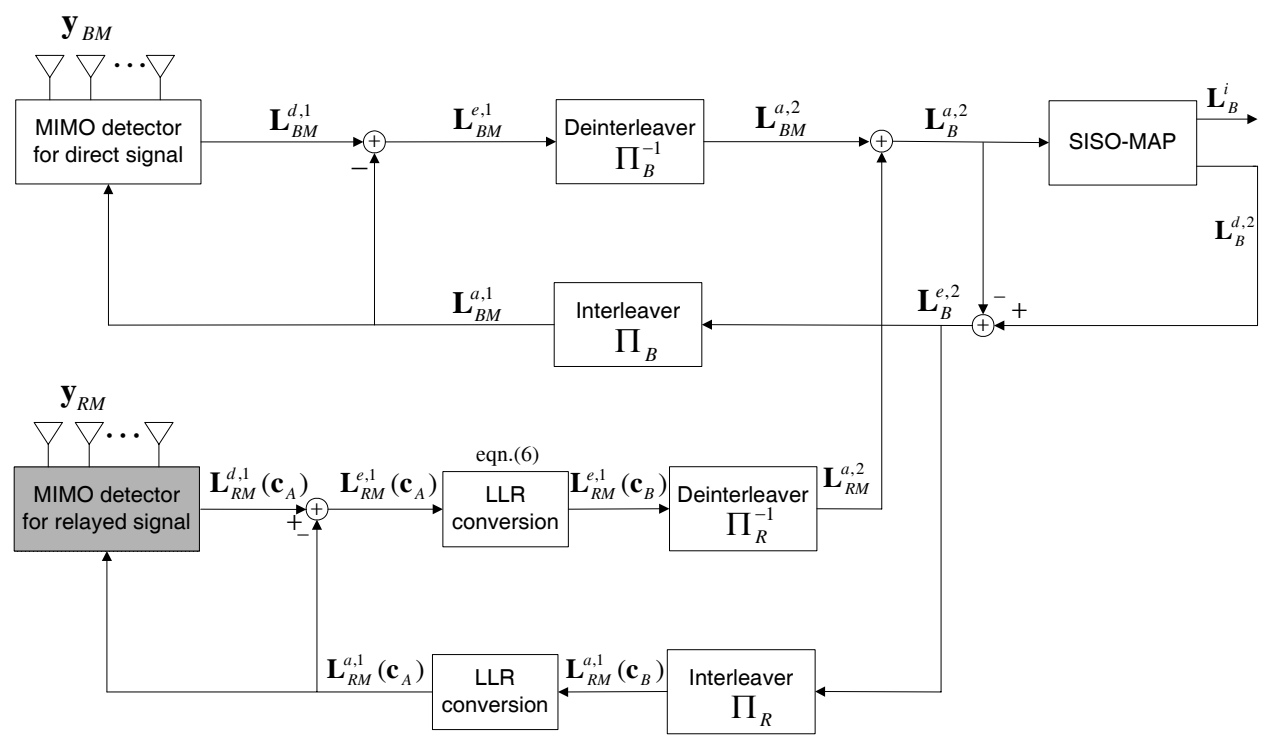

Fig. 4. Block diagram of the proposed decoding scheme at the MS

known at the MS. Based on the XOR operation, we change the signs of those elements of $\mathbf{L}_{R M}^{e, 1}\left(\mathbf{c}_{A}\right)$, whose corresponding bit values in $\mathbf{c}_{M}$ are 1 , formulated as:

$L_{R M}^{e, 1}\left(c_{B, k}\right)=\left\{\begin{array}{c}L_{R M}^{e, 1}\left(c_{A, k}\right), \text { if the } k \text { th element of } \mathbf{c}_{M} \text { is } 0 \\ -L_{R M}^{e, 1}\left(c_{A, k}\right), \text { if the } k \text { th element of } \mathbf{c}_{M} \text { is } 1\end{array}\right.$,

where $L_{R M}^{e, 1}\left(c_{A, k}\right)$ is the $k$ th element of $\mathbf{L}_{R M}^{e, 1}\left(\mathbf{c}_{A}\right)$, and $L_{R M}^{e, 1}\left(c_{B, k}\right)$ is the $k$ th element of the resultant LLR $\mathbf{L}_{R M}^{e, 1}\left(\mathbf{c}_{B}\right)$ for $\mathbf{c}_{B}$, which is used to obtain the input of the channel decoder.

When decoding $\mathbf{c}_{B}$, we also exploit the directly detected signal $\mathbf{y}_{B M}$ of (2) as shown in Fig. 4 in order to improve the attainable performance. The MIMO detector extracts the LLR $\mathbf{L}_{B M}^{e, 1}$ representing $\mathbf{c}_{B}$ from $\mathbf{y}_{B M}$, which is then interleaved and combined with $\mathbf{L}_{R M}^{a, 2}$, namely the interleaved version of the LLRs obtained from the relayed signal $\mathbf{y}_{R M}$ of Fig. 4. We note that the a-priori information is subtracted, before the a-posteriori LLRs are combined and forwarded to the channel decoder.

In practical relaying systems, the cyclic redundancy check (CRC) is adopted for error detection at the RS. If no errors are detected, the RS combines the estimated $\mathbf{c}_{B}$ and $\mathbf{c}_{M}$ using element-wise XOR operation into a composite packet and forwards it to both the BS and the MS, as seen in Fig. 1. However, if errors are detected, we assume that the RS forwards the bit stream, which may have decoding errors. In the forthcoming section, we consider two types of relaying strategies designed for mitigating the effect of erroneous forwarded streams, namely the HDF and SDF. Furthermore, we derive decoding algorithms for employment at the destination node, which are robust to decoding errors encountered at the RS. 


$$
\begin{aligned}
& \quad \sum_{\left\{\mathbf{c}_{A}^{l}, \Delta \mathbf{c}_{A}^{l}\right\}: c_{A, m}=1} d\left(\hat{\mathbf{x}}_{A}\right) p\left(\mathbf{c}_{A}^{l}, \Delta \mathbf{c}_{A}^{l}\right) \\
& =\sum_{\left\{\mathbf{c}_{A}^{l}, \Delta \mathbf{c}_{A}^{l}\right\}: c_{A, m}=1, c_{A, 1}=0, \Delta c_{A, 1}=0} d\left(\hat{\mathbf{x}}_{A}\right) p\left(\Delta c_{A, 1}=0\right) p\left(c_{A, 1}=0\right) \prod_{k=2}^{N_{t} M_{c}} p\left(\Delta c_{A, k}\right) p\left(c_{A, k}\right) \\
& +\sum_{\left\{\mathbf{c}_{A}^{l}, \Delta \mathbf{c}_{A}^{l}\right\}: c_{A, m}=1, c_{A, 1}=0, \Delta c_{A, 1}=1} d\left(\hat{\mathbf{x}}_{A}\right) p\left(\Delta c_{A, 1}=1\right) p\left(c_{A, 1}=0\right) \prod_{k=2}^{N_{t} M_{c}} p\left(\Delta c_{A, k}\right) p\left(c_{A, k}\right) \\
& +\sum_{\left\{\mathbf{c}_{A}^{l}, \Delta \mathbf{c}_{A}^{l}\right\}: c_{A, m}=1, c_{A, 1}=1, \Delta c_{A, 1}=0} d\left(\hat{\mathbf{x}}_{A}\right) p\left(\Delta c_{A, 1}=0\right) p\left(c_{A, 1}=1\right) \prod_{k=2}^{N_{t} M_{c}} p\left(\Delta c_{A, k}\right) p\left(c_{A, k}\right) \\
& +\sum_{\left\{\mathbf{c}_{A}^{l}, \Delta \mathbf{c}_{A}^{l}\right\}: c_{A, m}=1, c_{A, 1}=1, \Delta c_{A, 1}=1} d\left(\hat{\mathbf{x}}_{A}\right) p\left(\Delta c_{A, 1}=1\right) p\left(c_{A, 1}=1\right) \prod_{k=2}^{N_{t} M_{c}} p\left(\Delta c_{A, k}\right) p\left(c_{A, k}\right) .
\end{aligned}
$$

\section{Decoding Schemes for Network Coding Aided RELAYING}

\section{A. MIMO decoder for hard decision relaying}

In HDF relaying, the relay obtains the estimates of $\mathbf{c}_{B}$ or $\mathbf{c}_{M}$ by slicing the elements of the LLR vector $\mathbf{L}_{B R}^{d, 2}$ or $\mathbf{L}_{M R}^{d, 2}$, even if decoding errors occur. The estimated $\mathbf{c}_{B}$ and $\mathbf{c}_{M}$ values are combined to generate $\hat{\mathbf{c}}_{A}$, which is the estimate of $\mathbf{c}_{A}$, even if $\mathbf{c}_{B}$ or $\mathbf{c}_{M}$ contains errors. Considering this scenario, we derive the optimal decoding scheme for MIMO relaying systems.

We assume that the bit error rate of the composite packet $\hat{\mathbf{c}}_{A}$ is $q(<1 / 2)$, i.e. $q=d\left(\hat{\mathbf{c}}_{A}, \mathbf{c}_{A}\right) /\left(L N_{t} M_{c}\right)$, where $d(\cdot, \cdot)$ denotes the Hamming distance between two vectors. We also define $\hat{c}_{A, m}$ and $\hat{\mathbf{c}}_{A}^{l}$ as the $m$ th estimated coded bit and the estimated $\left(N_{t} M_{c} \times 1\right)$-element bit stream vector corresponding to $c_{A, m}$ and $\mathbf{c}_{A}^{l}$, respectively. Here, we express $\hat{c}_{A, m}$ and $\hat{\mathbf{c}}_{A}^{l}$ as

$$
\begin{aligned}
\hat{c}_{A, m} & =c_{A, m} \oplus \Delta c_{A, m}, \\
\hat{\mathbf{c}}_{A}^{l} & =\mathbf{c}_{A}^{l} \oplus \Delta \mathbf{c}_{A}^{l},
\end{aligned}
$$

where $\Delta c_{A, m} \in\{0,1\}$ and $\Delta \mathbf{c}_{A}^{l}$ represent the corresponding decoding errors in $c_{A, m}$ and $\mathbf{c}_{A}^{l}$, respectively. We define $\hat{\mathbf{x}}_{A}^{h}$ as the modulated signal vector of $\hat{\mathbf{c}}_{A}^{l}$. The modulated signal $\hat{\mathbf{x}}_{A}^{h}$ is transmitted and the corresponding received signals at the MS and the BS are formulated as

$$
\begin{aligned}
\mathbf{y}_{R M}^{h} & =\mathbf{H}_{R M} \hat{\mathbf{x}}_{A}^{h}+\mathbf{v}_{R M}, \\
\mathbf{y}_{R B}^{h} & =\mathbf{H}_{R B} \hat{\mathbf{x}}_{A}^{h}+\mathbf{v}_{R B},
\end{aligned}
$$

where $\mathbf{H}_{R M}$ and $\mathbf{H}_{R B}$ denote the $\left(N_{r} \times N_{t}\right)$-element complex-valued channel matrices, while $\mathbf{v}_{R M}$ and $\mathbf{v}_{R B}$ are the $\left(N_{r} \times 1\right)$-element Gaussian distributed noise vectors of $\mathbf{v}_{R M}, \mathbf{v}_{R B} \sim \mathcal{C N}\left(\mathbf{0}_{N_{r}}, \sigma_{v}^{2} \mathbf{I}_{N_{r}}\right)$.

Hereafter, we will derive the optimal MIMO decoder for the DL signal of the HDF system. The BS's decoder of the UL signal can be readily obtained from the decoder of the DL at the MS as a benefit of the NCR system's symmetric structure.

For the signal directly transmitted from the BS, the conventional MIMO detector of [15], [16] is applied without any modification, because it does not contain the RS's decision error hosted by $\Delta \mathbf{c}_{A}^{l}$. Therefore, we only have to modify the MIMO detection block of the RS seen in Fig. 4 by considering the decoding error vector $\Delta \mathbf{c}_{A}^{l}$. The LLR of $c_{A, m}$ is formulated as

$$
\begin{aligned}
& L_{R M}^{d, 1}\left(c_{A, m}\right)= \\
& \log \frac{\sum_{\left\{\mathbf{c}_{A}^{l}, \Delta \mathbf{c}_{A}^{l}\right\}: c_{A, m}=1} e^{-\left\|\mathbf{y}_{R M}^{h}-\mathbf{H}_{R M} \hat{\mathbf{x}}_{A}\right\|^{2} / \sigma^{2}} p\left(\mathbf{c}_{A}^{l}, \Delta \mathbf{c}_{A}^{l}\right)}{\sum_{\left\{\mathbf{c}_{A}^{l}, \Delta \mathbf{c}_{A}^{l}\right\}: c_{A, m}=0} e^{-\left\|\mathbf{y}_{R M}^{h}-\mathbf{H}_{R M} \hat{\mathbf{x}}_{A}\right\|^{2} / \sigma^{2}} p\left(\mathbf{c}_{A}^{l}, \Delta \mathbf{c}_{A}^{l}\right)},
\end{aligned}
$$

where $\hat{\mathbf{x}}_{A}$ is a trial of $\hat{\mathbf{x}}_{A}^{h}$ corresponding to $\left\{\mathbf{c}_{A}^{l}, \Delta \mathbf{c}_{A}^{l}\right\}$ and $p\left(\mathbf{c}_{A}^{l}, \Delta \mathbf{c}_{A}^{l}\right)$ is the joint probability of $\mathbf{c}_{A}^{l}$ and $\Delta \mathbf{c}_{A}^{l}$. Furthermore, $\|\cdot\|$ indicates the Frobenius norm of a matrix. Assuming that the elements of $\mathbf{c}_{A}^{l}$ and $\Delta \mathbf{c}_{A}^{l}$ are mutually independent thanks to the random interleaver at the transmitter, respectively, $p\left(\mathbf{c}_{A}^{l}, \Delta \mathbf{c}_{A}^{l}\right)$ can be written as

$$
p\left(\mathbf{c}_{A}^{l}, \Delta \mathbf{c}_{A}^{l}\right)=\prod_{k=1}^{N_{t} M_{c}} p\left(\Delta c_{A, k}\right) p\left(c_{A, k}\right)
$$

Since the error rate of $\mathbf{c}_{A}$ is $q, p\left(\Delta c_{A, k}\right)$ becomes

$$
p\left(\Delta c_{A, k}\right)=\left\{\begin{array}{cc}
1-q, & \text { if } \Delta c_{A, k}=0 \\
q, & \text { if } \Delta c_{A, k}=1
\end{array} .\right.
$$

Then, the likelihood function for $c_{A, m}=1$ in (11) is expressed as

$$
\begin{aligned}
& \sum_{\left\{\mathbf{c}_{A}^{l}, \Delta \mathbf{c}_{A}^{l}\right\}: c_{A, m}=1} e^{-\left\|\mathbf{y}_{R M}^{h}-\mathbf{H}_{R M} \hat{\mathbf{x}}_{A}\right\|^{2} / \sigma^{2}} p\left(\mathbf{c}_{A}^{l}, \Delta \mathbf{c}_{A}^{l}\right) \\
= & \sum_{\left\{\mathbf{c}_{A}^{l}, \Delta \mathbf{c}_{A}^{l}\right\}: c_{A, m}=1} d\left(\hat{\mathbf{x}}_{A}\right) \prod_{k=1}^{N_{t} M_{c}} p\left(\Delta c_{A, k}\right) p\left(c_{A, k}\right),
\end{aligned}
$$

where $d\left(\hat{\mathbf{x}}_{A}\right)=e^{-\left\|\mathbf{y}_{R M}^{h}-\mathbf{H}_{R M} \hat{\mathbf{x}}_{A}\right\|^{2} / \sigma^{2}}$.

We can divide (14) into two terms, corresponding to $c_{A, 1}=$ 


$$
\begin{aligned}
L_{R M}^{d, 1}\left(c_{A, m}\right) & =\log \frac{p\left(c_{A, m}=1\right)}{p\left(c_{A, m}=0\right)} \\
& +\log \frac{(1-q) \sum_{\hat{\mathbf{c}}_{A}^{l}: \hat{c}_{A, m}=1} d\left(\hat{\mathbf{x}}_{A}\right) \prod_{k=1, k \neq m}^{N_{t} M_{c}} p\left(\hat{c}_{A, k}\right)+q \sum_{\hat{\mathbf{c}}_{A}^{l}: \hat{c}_{A, m}=0} d\left(\hat{\mathbf{x}}_{A}\right) \prod_{k=1, k \neq m}^{N_{t} M_{c}} p\left(\hat{c}_{A, k}\right)}{(1-q) \sum_{\hat{\mathbf{c}}_{A}^{l}: \hat{c}_{A, m}=0} d\left(\hat{\mathbf{x}}_{A}\right) \prod_{k=1, k \neq m}^{N_{t} M_{c}} p\left(\hat{c}_{A, k}\right)+q \sum_{\hat{\mathbf{c}}_{A}^{l}: \hat{c}_{A, m}=1} d\left(\hat{\mathbf{x}}_{A}\right) \prod_{k=1, k \neq m}^{N_{t} M_{c}} p\left(\hat{c}_{A, k}\right)} .
\end{aligned}
$$

$$
L_{R M}^{e, 1}\left(c_{A, m}\right)=\log \frac{(1-q) \sum_{\hat{\mathbf{c}}_{A}^{l}: \hat{c}_{A, m}=1} d\left(\hat{\mathbf{x}}_{A}\right) \prod_{k=1, k \neq m}^{N_{t} M_{c}} p\left(\hat{c}_{A, k}\right)+q \sum_{\hat{\mathbf{c}}_{A}^{l}: \hat{c}_{A, m}=0} d\left(\hat{\mathbf{x}}_{A}\right) \prod_{k=1, k \neq m}^{N_{t} M_{c}} p\left(\hat{c}_{A, k}\right)}{(1-q) \sum_{\hat{\mathbf{c}}_{A}^{l}: \hat{c}_{A, m}=0} d\left(\hat{\mathbf{x}}_{A}\right) \prod_{k=1, k \neq m}^{N_{t} M_{c}} p\left(\hat{c}_{A, k}\right)+q \sum_{\hat{\mathbf{c}}_{A}^{l}: \hat{c}_{A, m}=1} d\left(\hat{\mathbf{x}}_{A}\right) \prod_{k=1, k \neq m}^{N_{t} M_{c}} p\left(\hat{c}_{A, k}\right)} .
$$

0 and $c_{A, 1}=1$ in order to obtain

$$
\begin{aligned}
& \sum_{\hat{\mathbf{x}}_{A}: c_{A, m}=1} d\left(\hat{\mathbf{x}}_{A}\right) p\left(\mathbf{c}_{A}^{l}, \Delta \mathbf{c}_{A}^{l}\right) \\
= & \sum_{\left\{\mathbf{c}_{A}^{l}, \Delta \mathbf{c}_{A}^{l}\right\}: c_{A, m}=1, c_{A, 1}=0} d\left(\hat{\mathbf{x}}_{A}\right) p\left(\Delta c_{A, 1}\right) p\left(c_{A, 1}=0\right) \\
& \cdot \prod_{k=2}^{N_{t} M_{c}} p\left(\Delta c_{A, k}\right) p\left(c_{A, k}\right) \\
& +\sum_{\left\{\mathbf{c}_{A}^{l}, \Delta \mathbf{c}_{A}^{l}\right\}: c_{A, m}=1, c_{A, 1}=1} d\left(\hat{\mathbf{x}}_{A}\right) p\left(\Delta c_{A, 1}\right) p\left(c_{A, 1}=1\right) \\
& \cdot \prod_{k=2}^{N_{t} M_{c}} p\left(\Delta c_{A, k}\right) p\left(c_{A, k}\right),
\end{aligned}
$$

which can be further divided into four terms with respect to $\Delta c_{A, 1}$, yielding (16). We note that for both $\left\{c_{A, 1}=\right.$ $\left.0, \Delta c_{A, 1}=0\right\}$ and $\left\{\Delta c_{A, 1}=1, c_{A, 1}=1\right\}, \hat{c}_{A, 1}$ is constant (i.e., $\hat{c}_{A, 1}=0$ ). Therefore, for $\left\{c_{A, 1}=0, \Delta c_{A, 1}=0\right\}$ and $\left\{\Delta c_{A, 1}=1, c_{A, 1}=1\right\}, \mathbf{x}_{A}$ has the same value and we can combine the first and last terms in (16). In a similar manner, we combine the second and third terms and use (13) to obtain

$$
\begin{aligned}
& \sum_{\left\{\mathbf{c}_{A}^{l}, \Delta \mathbf{c}_{A}^{l}\right\}: c_{A, m}=1} d\left(\hat{\mathbf{x}}_{A}\right) p\left(\mathbf{c}_{A}^{l}, \Delta \mathbf{c}_{A}^{l}\right) \\
& =\sum_{\left\{\mathbf{c}_{A, 2}^{l}, \Delta \mathbf{c}_{A, 2}^{l}, \hat{c}_{A, 1}\right\}: c_{A, m}=1, \hat{c}_{A, 1}=0} d\left(\hat{\mathbf{x}}_{A}\right)\left\{(1-q) p\left(c_{A, 1}=0\right)\right. \\
& \left.+q p\left(c_{A, 1}=1\right)\right\} \prod_{k=2}^{N_{t} M_{c}} p\left(\Delta c_{A, k}\right) p\left(c_{A, k}\right) \\
& +\sum_{\left\{\mathbf{c}_{A, 2}^{l}, \Delta \mathbf{c}_{A, 2}^{l}, \hat{c}_{A, 1}\right\}: c_{A, m}=1, \hat{c}_{A, 1}=1}^{N_{t} M_{c}} d\left(\hat{\mathbf{x}}_{A}\right)\left\{q p\left(c_{A, 1}=0\right)\right. \\
& \left.+(1-q) p\left(c_{A, 1}=1\right)\right\} \prod_{k=2} p\left(\Delta c_{A, k}\right) p\left(c_{A, k}\right),
\end{aligned}
$$

where we have $\mathbf{c}_{A, k}^{l}=\left\{c_{A, k}, c_{A, k+1}, \cdots, c_{A, N_{t} M_{c}}\right\}$ and $\Delta \mathbf{c}_{A, k}^{l}=\left\{\Delta c_{A, k}, \Delta c_{A, k+1}, \cdots, c_{A, N_{t} M_{c}}\right\}$. Here, it is worth noting that the a-priori probability of $\hat{c}_{A, k}$ is expressed as

$$
p\left(\hat{c}_{A, k}\right)=\left\{\begin{array}{l}
(1-q) p\left(c_{A, k}=0\right)+q p\left(c_{A, k}=1\right), \text { if } \hat{c}_{A, k}=0 \\
q p\left(c_{A, k}=0\right)+(1-q) p\left(c_{A, k}=1\right), \text { if } \hat{c}_{A, k}=1
\end{array} .\right.
$$

Employing (17) and (18) can be simplified as

$$
\begin{aligned}
& \sum_{\left\{\mathbf{c}_{A}^{l}, \Delta \mathbf{c}_{A}^{l}\right\}: c_{A, m}=1} d\left(\hat{\mathbf{x}}_{A}\right) p\left(\mathbf{c}_{A}^{l}, \Delta \mathbf{c}_{A}^{l}\right)= \\
& \sum_{\left\{\mathbf{c}_{A, 2}^{l}, \Delta \mathbf{c}_{A, 2}^{l}, \hat{c}_{A, 1}\right\}: c_{A, m}=1} d\left(\hat{\mathbf{x}}_{A}\right) p\left(\hat{c}_{A, 1}\right) \prod_{k=2}^{N_{t} M_{c}} p\left(\Delta c_{A, k}\right) p\left(c_{A, k}\right) .
\end{aligned}
$$

Applying similar operations to those in (15)-(19) for $\left\{c_{A, 2}, c_{A, 3}, \cdots, c_{A, N_{t} M_{c}}\right\}$ except for $c_{A, m}$, we arrive at

$$
\begin{aligned}
& \sum_{\left\{\mathbf{c}_{A}^{l}, \Delta \mathbf{c}_{A}^{l}\right\}: c_{A, m}=1} d\left(\hat{\mathbf{x}}_{A}\right) p\left(\mathbf{c}_{A}^{l}, \Delta \mathbf{c}_{A}^{l}\right) \\
& =\sum_{\left\{c_{A, m}, \Delta c_{A, m}, \hat{\mathbf{c}}_{A, m}^{l}\right\}: c_{A, m}=1} d\left(\hat{\mathbf{x}}_{A}\right) p\left(\Delta c_{A, m}\right) p\left(c_{A, m}=1\right) \\
& \cdot \prod_{k=1, k \neq m}^{N_{t} M_{c}} p\left(\hat{c}_{A, k}\right) \\
& =p\left(c_{A, m}=1\right)\left\{(1-q) \sum_{\hat{\mathbf{c}}_{A}^{l}: \hat{c}_{A, m}=1}^{N_{t} M_{c}} d\left(\hat{\mathbf{x}}_{A}\right) \prod_{k=1, k \neq m}^{N_{t} M_{c}} p\left(\hat{c}_{A, k}\right)\right. \\
& \quad+q \sum_{\hat{\mathbf{c}}_{A}^{l}: \hat{c}_{A, m}=0} d\left(\hat{\mathbf{x}}_{A}\right) \prod_{k=1, k \neq m}^{\left.\left.N_{c_{A, k}}\right)\right\},}
\end{aligned}
$$

where $\hat{\mathbf{c}}_{A, \bar{m}}^{l}$ denotes the estimated bit stream vector obtained by excluding $\hat{c}_{A, m}$ from $\hat{\mathbf{c}}_{A}$. In a similar manner, we simplify the likelihood function for $c_{A, m}=0$ and obtain the LLR for $c_{A, m}$, yielding (21). Upon excluding the a-priori part, we obtain the extrinsic information as (22).

Some remarks concerning the decoder proposed for the HDF system are provided below.

1) The original LLR formula of (11) considers $\Delta \mathbf{c}_{A}^{l}$ as well as $\mathbf{c}_{A}^{l}$ in order to compute the a-posteriori probability. Therefore, it is necessary to check a total of $2^{2 N_{t} M_{c}}$ hypotheses. However, in the simplified LLR formula of (21), we employ the probability of $\hat{c}_{A, k}$ instead of the probabilities of $c_{A, k}$ and $\Delta c_{A, k}$, hence we only check $2^{N_{t} M_{c}}$ hypotheses, which leads to a factor of $2^{N_{t} M_{c}}$ reduction of the computational load.

2) We note that we need $p\left(\hat{c}_{A, k}\right)$ for calculating the extrinsic LLR of (22) instead of $p\left(c_{A, k}\right)$. Therefore, we transform the a-priori information of $c_{A, k}$ to the a-priori information of $\hat{c}_{A, k}$ by using (18), before commencing the calculation of (22). 
3) $\begin{array}{llr}L_{R M}^{e, 1}\left(c_{A, m}\right) \text { of } \quad(22) \quad \text { has the } & \text { maxi- } \\ \text { mum value of } \log \{(1-q) / q\} & \text { when } \\ \sum_{\hat{\mathbf{c}}_{A}^{l}: \hat{c}_{A, m}=1} d\left(\hat{\mathbf{x}}_{A}\right) \prod_{k=1, k \neq m}^{N_{t} M_{c}} p\left(\hat{c}_{A, k}\right) & \gg \\ \sum_{\hat{\mathbf{c}}_{A}^{l}: \hat{c}_{A, m}=0} d\left(\hat{\mathbf{x}}_{A}\right) \prod_{k=1, k \neq m}^{N_{t} M_{c}} p\left(\hat{c}_{A, k}\right) \text {. By } & \text { contrast, } \\ \text { the minimum value of } \log \{q /(1-q)\} & \text { occurs, } \\ \text { when } \sum_{\hat{\mathbf{c}}_{A}^{l}: \hat{c}_{A, m}=1} d\left(\hat{\mathbf{x}}_{A}\right) \prod_{k=1, k \neq m}^{N_{t} M_{c}} p\left(\hat{c}_{A, k}\right) & \ll \\ \sum_{\hat{\mathbf{c}}_{A}^{l}: \hat{c}_{A, m}=0} d\left(\hat{\mathbf{x}}_{A}\right) \prod_{k=1, k \neq m}^{N_{t} M_{c}} p\left(\hat{c}_{A, k}\right) .\end{array}$

\section{B. MIMO decoder for soft decision relaying}

The HDF generally imposes a lower computational complexity than the SDF at the cost of a performance degradation, because it discards the soft information by slicing the signal. In this subsection, we derive a MIMO decoder for the SDF system for the sake of attaining a better performance. We assume that the RS transmits the expectation values of the symbols [14]. For example, when BPSK modulation is assumed, the $k$ th element of $\hat{\mathbf{x}}_{A}^{s}$ is formulated as

$$
\hat{x}_{A, k}^{s}=p\left(c_{A, k}=1 \mid \mathbf{y}_{M R}, \mathbf{y}_{B R}\right)-p\left(c_{A, k}=0 \mid \mathbf{y}_{M R}, \mathbf{y}_{B R}\right),
$$

where $\hat{\mathbf{x}}_{A}^{s}$ is the soft estimate of $\mathbf{x}_{A}$. More explicitly, at the RS, we employ the IDD scheme of Fig. 3 to compute the LLRs of $\mathbf{c}_{B}$ and $\mathbf{c}_{M}$. In SDF, the composite bit $c_{A, k}$ is estimated from LLRs of $\mathbf{c}_{B}$ and $\mathbf{c}_{M}$. We denote the LLRs of $c_{B, k}$ and $c_{M, k}$ as $L_{B, k}$ and $L_{M, k}$, respectively, where $c_{B, k}$ and $c_{M, k}$ are elements in $\mathbf{c}_{B}$ and $\mathbf{c}_{M}$, determining $c_{A, k}=c_{B, k} \oplus c_{M, k}$. The probability of a bit $c$ is expressed as $p(c=1)=1 /(1+$ $\left.e^{-L}\right)$ and $p(c=0)=e^{-L} /\left(1+e^{-L}\right)$, respectively, when $L$ is the corresponding LLR. Therefore, the probability of $c_{A, k}$ becomes

$$
\begin{aligned}
& p\left(c_{A, k}=0 \mid \mathbf{y}_{M R}, \mathbf{y}_{B R}\right)= \\
& \frac{1}{1+e^{-L_{B, k}}} \cdot \frac{e^{-L_{M, k}}}{1+e^{-L_{M, k}}}+\frac{e^{-L_{B, k}}}{1+e^{-L_{B, k}}} \cdot \frac{1}{1+e^{-L_{M, k}}}, \\
& p\left(c_{A, k}=1 \mid \mathbf{y}_{M R}, \mathbf{y}_{B R}\right)= \\
& \frac{1}{1+e^{-L_{B, k}}} \cdot \frac{1}{1+e^{-L_{M, k}}}+\frac{e^{-L_{B, k}}}{1+e^{-L_{B, k}}} \cdot \frac{e^{-L_{M, k}}}{1+e^{-L_{M, k}}} .
\end{aligned}
$$

Using (24) and (25), we are capable of obtaining symbol probabilities and computing the soft estimate of a symbol as exemplified in (23).

By transmitting the expectation value of a symbol rather than the sliced value, we minimize the mean squared error of the relayed signals and preserve the soft information. In [14], the error of $\hat{x}_{A, k}^{s}$ was modeled as a Gaussian distributed random variable. However, to obtain a more accurate error model, we divide the error into two terms: the hard-decision error and the soft-decision error. The error vector $\Delta \mathrm{x}_{A}=$ $\hat{\mathbf{x}}_{A}^{s}-\mathbf{x}_{A}$ is expressed as

$$
\Delta \mathbf{x}_{A}=\Delta \mathbf{x}_{A}^{h}+\Delta \mathbf{x}_{A}^{s},
$$

where $\Delta \mathbf{x}_{A}^{h}=M\left(\hat{\mathbf{c}}_{A}^{l}\right)-\mathbf{x}_{A}$ is the hard-valued error vector and $\Delta \mathbf{x}_{A}^{s}$ represents the soft-valued error vector. Here, $M(\cdot)$ denotes the constellation mapper. It is worthwhile noting that $\Delta \mathbf{x}_{A}^{h}$ is the same as the symbol error vector in the HDF system. We assume that the elements of $\Delta \mathbf{x}_{A}^{s}$ are independent zero-mean Gaussian distributed random variables, but they have different variances depending on the specific value of
$\Delta \mathbf{x}_{A}^{h}$. Explicitly, we assume that the variance of the $k$ th element of $\Delta \mathbf{x}_{A}^{s}$ is $\sigma_{e, 1}^{2}$ for $\Delta x_{A, k}^{h}=0$, where $\Delta x_{A, k}^{h}$ is the $k$ th element of $\Delta \mathbf{x}_{A}^{h}$. Furthermore, the variance of the $k$ th element of $\Delta \mathbf{x}_{A}^{s}$ is denoted as $\sigma_{e, 2}^{2}$ for $\Delta x_{A}^{h} \neq 0$, which corresponds to the case when a hard-decision based error occurs. Generally, $\sigma_{e, 2}^{2}$ is higher than $\sigma_{e, 1}^{2}$ because the expectation value of a symbol is typically around the decision boundary when the hard-valued error occurs.

The received signal at the MS is expressed as

$$
\mathbf{y}_{R M}^{s}=\mathbf{H}_{R M} \hat{\mathbf{x}}_{A}^{s}+\mathbf{v}_{R M} .
$$

Considering that we have

$$
\hat{\mathbf{x}}_{A}^{s}=\mathbf{x}_{A}+\Delta \mathbf{x}_{A}^{h}+\Delta \mathbf{x}_{A}^{s},
$$

(27) can be rewritten as

$$
\mathbf{y}_{R M}^{s}=\mathbf{H}_{R M}\left(\mathbf{x}_{A}+\Delta \mathbf{x}_{A}^{h}\right)+\mathbf{H}_{R M} \Delta \mathbf{x}_{A}^{s}+\mathbf{v}_{R M} .
$$

Furthermore, exploiting that $\hat{\mathbf{x}}_{A}^{h}=\mathbf{x}_{A}+\Delta \mathbf{x}_{A}^{h}$, we obtain

$$
\mathbf{y}_{R M}^{s}=\mathbf{H}_{R M} \hat{\mathbf{x}}_{A}^{h}+\mathbf{H}_{R M} \Delta \mathbf{x}_{A}^{s}+\mathbf{v}_{R M},
$$

where $\mathbf{H}_{R M} \Delta \mathbf{x}_{A}^{s}$ can be regarded as an interference term. Comparing (9) and (30), we can see that the only difference between them is that the interference term of $\mathbf{H}_{R M} \Delta \mathbf{x}_{A}^{s}$ is added in (30). Hence we can apply the proposed MIMO decoder in Section III-A to the SDF system by considering $\tilde{\mathbf{v}}=\mathbf{H}_{R M} \Delta \mathbf{x}_{A}^{s}+\mathbf{v}_{R M}$ as a new interference-plus-noise term. Since $\Delta \mathbf{x}_{A}^{s}$ and $\mathbf{v}_{R M}$ are Gaussian distributed random vectors, we know that $\tilde{\mathbf{v}}$ is also a Gaussian distributed random vector of $\tilde{\mathbf{v}} \sim \mathcal{C N}\left(\mathbf{0}_{N_{r}}, \mathbf{R}_{\tilde{v}}\right)$, where $\mathbf{R}_{\tilde{v}}=\sigma_{v}^{2} \mathbf{I}_{N_{r}}+\mathbf{H}_{R M} \boldsymbol{\Lambda} \mathbf{H}_{R M}^{H}$. Here, $(\cdot)^{H}$ denotes the complex conjugate transpose of a matrix and $\Lambda$ is a diagonal matrix, where the $k$ th element is $\sigma_{e, 1}^{2}$ if $\Delta x_{A, k}^{h}=0$, or $\sigma_{e, 2}^{2}$ if $\Delta x_{A, k}^{h} \neq 0$.

Therefore, the LLR of $c_{A, m}$ can be computed as

$$
\begin{aligned}
& L_{R M}^{d, 1}\left(c_{A, m}\right) \\
& =\log \frac{\sum_{\left\{\mathbf{c}_{A}^{l}, \Delta \mathbf{c}_{A}^{l}\right\}: c_{A, m}=1} d\left(\hat{\mathbf{x}}_{A}, \Delta \mathbf{x}_{A}^{h}\right) p\left(\mathbf{c}_{A}^{l}, \Delta \mathbf{c}_{A}^{l}\right)}{\sum_{\left\{\mathbf{c}_{A}^{l}, \Delta \mathbf{c}_{A}^{l}\right\}: c_{A, m}=0} d\left(\hat{\mathbf{x}}_{A}, \Delta \mathbf{x}_{A}^{h}\right) p\left(\mathbf{c}_{A}^{l}, \Delta \mathbf{c}_{A}^{l}\right)},
\end{aligned}
$$

where $d\left(\hat{\mathbf{x}}_{A}, \Delta \mathbf{x}_{A}^{h}\right)=e^{-\left(\mathbf{y}_{R M}^{s}-\mathbf{H}_{R M} \hat{\mathbf{x}}_{A}\right)^{H} \mathbf{R}_{\tilde{v}}^{-1}\left(\mathbf{y}_{R M}^{s}-\mathbf{H}_{R M} \hat{\mathbf{x}}_{A}\right) / 4}$. Notice that $\mathbf{R}_{\tilde{v}}$ depends on $\Delta \mathbf{x}_{A}^{h}$. Since $d\left(\hat{\mathbf{x}}_{A}, \Delta \mathbf{x}_{A}^{h}\right)$ is a function of both $\Delta \mathbf{x}_{A}^{h}$ as well as $\hat{\mathbf{x}}_{A}$, we are unable to simplify (31) as we did in Section III-A. Furthermore, it is very difficult to estimate $\sigma_{e, 2}^{2}$ accurately, because only a few bit errors occur when we decode a single codeword in the high signal-to-noise ratio (SNR) region. More explicitly, we should estimate $\sigma_{e, 2}^{2}$ for a low number of samples, which are likely to be decoding errors in a codeword. Therefore, the resultant estimate typically has a large error, which results in a performance degradation. Owing to these reasons, the proposed soft error model has to be modified in order to reduce the computational complexity imposed and improve the attainable performance, we combine $\sigma_{e, 1}^{2}$ and $\sigma_{e, 2}^{2}$ into a single value $\sigma_{e}^{2}$, which is defined as the variance of the entries in $\Delta \mathrm{x}_{A}^{s}$, regardless of the specific values of $\Delta \mathrm{x}_{A}^{h}$. Accordingly, the covariance matrix of the soft error is rewritten as $\mathbf{R}_{\tilde{v}}=\sigma_{v}^{2} \mathbf{I}_{N_{r}}+\sigma_{e}^{2} \mathbf{H}_{R M} \mathbf{H}_{R M}^{H}$, which does not depend on $\Delta \mathbf{x}_{A}^{h}$. This allows us to simplify the computation of the extrinsic information as we did in (11)-(22) of Section 
III-A since $\mathbf{R}_{\tilde{v}}$ does not depend on $\Delta \mathbf{x}_{A}^{h}$. Explicitly, when computing $L_{R M}^{d, 1}\left(c_{A, m}\right)$ of (31), all we have to do is to change $d\left(\hat{\mathbf{x}}_{A}\right)$ in (21) to

$$
d\left(\hat{\mathbf{x}}_{A}\right)=e^{-\left(\mathbf{y}_{R M}^{s}-\mathbf{H}_{R M} \hat{\mathbf{x}}_{A}\right)^{H} \mathbf{R}_{\tilde{v}}^{-1}\left(\mathbf{y}_{R M}^{s}-\mathbf{H}_{R M} \hat{\mathbf{x}}_{A}\right) / 4} .
$$

We note that the likelihood function of (32) considers the effects of both soft-decision errors as well as of the Additive White Gaussian Noise (AWGN), while the HDF decoder only considers the AWGN. When the soft-decision error of a codeword is low, the likelihood function of (32) is reduced to that of the HDF system.

Consequently, we can compute the extrinsic information for the SDF system by using (22) in conjunction with the likelihood function of (32).

\section{Parameter estimation}

To apply the proposed decoders described in the previous subsections, we need the knowledge of the bit error ratio $q$ as well as the the variance of the soft error $\sigma_{e}^{2}$. These parameters are estimated at the RS and forwarded to both the BS and the MS. In this subsection, we derive methods to estimate $q$ and $\sigma_{e}^{2}$.

We employ (24) and (25) to estimate $q$. Since the specific logical bit value having a higher probability becomes the estimate of the coded bit in HDF, the error probability of $c_{A, k}$ is formulated as

$$
\begin{array}{r}
p_{e}\left(c_{A, k}\right)=\min \left\{p\left(c_{A, k}=0 \mid \mathbf{y}_{M R}, \mathbf{y}_{B R}\right),\right. \\
\left.p\left(c_{A, k}=1 \mid \mathbf{y}_{M R}, \mathbf{y}_{B R}\right)\right\} .
\end{array}
$$

Therefore, $q$ is estimated by averaging $p_{e}\left(c_{A, k}\right)$ over the entire codeword of length $T N_{t} M_{c}$.

By contrast, to obtain the estimate of $\sigma_{e}^{2}$ in SDF, we first compute $\hat{x}_{A, k}^{h}$, which is the hard-decision estimate of the $k$ th element of $\mathbf{x}_{A}$. The corresponding soft error $\Delta x_{A, k}^{s}$ is the difference between the soft-decision estimate $\hat{x}_{A, k}^{s}$ and the hard-decision estimate $\hat{x}_{A, k}^{h}$ as seen in (28). Hence the estimate of $\sigma_{e}^{2}$ is calculated by averaging $\left|\hat{x}_{A, k}^{s}-\hat{x}_{A, k}^{h}\right|^{2}$ over the entire codeword.

\section{Simulation Results}

Computer simulations have been performed to characterise the proposed decoding algorithms. We employed a turbo code having the rate $R=1 / 2$ and length of $T N_{t} M_{c}=1024$, which is constituted by two recursive systematic convolutional (RSC) codes with the octal generators $(7,5)$. Throughout our simulations, we used QPSK signaling and the exact log-MAP channel decoding algorithm at the receiver. The number of decoding iterations in the turbo channel decoder was set to five. We have assumed that all the elements of MIMO channel matrices are independent and identically distributed (i.i.d.) zero-mean complex Gaussian random variables having a variance of $1 / 2$ per dimension, which are fixed in a TS. We define the SNR as the ratio of the average power per information bit arriving at the receiver to the spectral density of the noise. In the IDD of the MIMO receiver, all possible symbol combinations are considered in the computation of (22). The number of MIMO detection/decoding iterations was selected to be four.

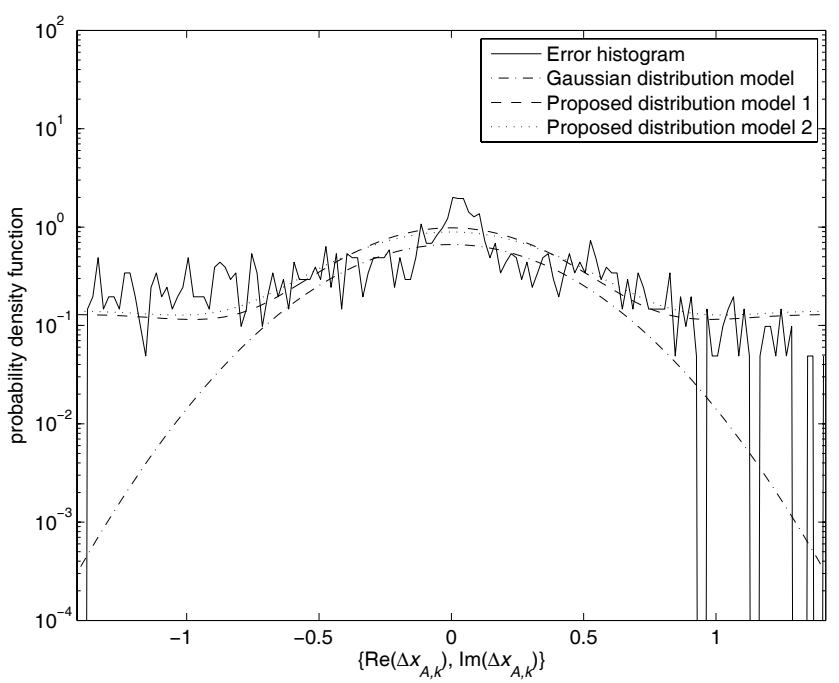

Fig. 5. An example histogram of the error signal and its distribution models using $N_{t}=2, N_{r}=2, \gamma_{M R}=2 \mathrm{~dB}, \gamma_{d i f f}=0 \mathrm{~dB}$. The parameters $\sigma_{e, 1}^{2}$, $\sigma_{e, 2}^{2}$, and $\sigma_{e}^{2}$ are estimated as $0.107,0.204$, and 0.130 , respectively.

The SNRs of the paths arriving from the BS and the MS to the RS are assumed to be the same and are denoted as $\gamma_{R S}$. This implies that the RS is somewhere half-way between the BS and MS, where the associated path loss and fading parameters are the same, although in practice the path loss between the BS and the RS may be expected to be lower than that between the RS and MS. Similarly, we assume that $\gamma_{M R}$, the SNR of the path from the RS to the MS, is the same as $\gamma_{R S}$. The SNR of the direct link from the BS to the MS is denoted as $\gamma_{B M}$. We also assume that the relay channel has the same or a higher SNR compared to the direct channel (i.e. $\left.\gamma_{M R} \geq \gamma_{B M}\right)$ and we denote the ratio between these SNRs as $\gamma_{\text {diff }}(>1)$.

Fig. 5 shows an example of the experimentally recorded error histogram and two other distributions modeling the realvalued error $\left\{\operatorname{Re}\left(\Delta x_{A, k}\right), \operatorname{Im}\left(\Delta x_{A, k}\right\}\right)$ for a single simulation run of SDF using $N_{t}=N_{r}=2, \gamma_{M R}=2 \mathrm{~dB}, \gamma_{\text {diff }}=0$ $\mathrm{dB}$, where $\Delta x_{A, k}=\Delta x_{A, k}^{h}+\Delta x_{A, k}^{s}$. We observe in Fig. 5 that the Gaussian distribution does not match well to the actual distribution of errors. Especially, for high error values, the Gaussian distribution tends to zero, while the experimental histogram indicates relatively high probabilities even for error values in excess of 0.5 . Hence here we propose a more accurate error error model as the combination of soft and hard errors, which is expressed as

$$
\begin{aligned}
& f\left(\Delta x_{A, k}\right)=(1-q) \cdot g\left(\Delta x_{A, k} \mid 0, \sigma_{e, 1}^{2}\right) \\
& +q \cdot g\left(\Delta x_{A, k} \mid \sqrt{2}, \sigma_{e, 2}^{2}\right)+q \cdot g\left(\Delta x_{A, k} \mid-\sqrt{2}, \sigma_{e, 2}^{2}\right), \\
& -\sqrt{2} \leq \Delta x_{A, k} \leq \sqrt{2},
\end{aligned}
$$

where $g\left(x \mid m, \sigma^{2}\right)=\frac{1}{\sqrt{2 \pi \sigma^{2}}} e^{|x-m|^{2} / 2 \sigma^{2}}$. This model, which is referred to as the proposed distribution model 1, was also plotted in Fig. 5. The error probability $q$ is estimated as described in Section III-C. The error variances $\sigma_{e, 1}^{2}$ and $\sigma_{e, 2}^{2}$ are obtained by averaging $\left(\Delta x_{A, k}^{s}\right)^{2}$ when we have $\Delta x_{A, k}^{h}=0$ and $\Delta x_{A, k}^{h} \neq 0$, respectively, even though in practical systems, this estimation method is almost infeasible, because we cannot 


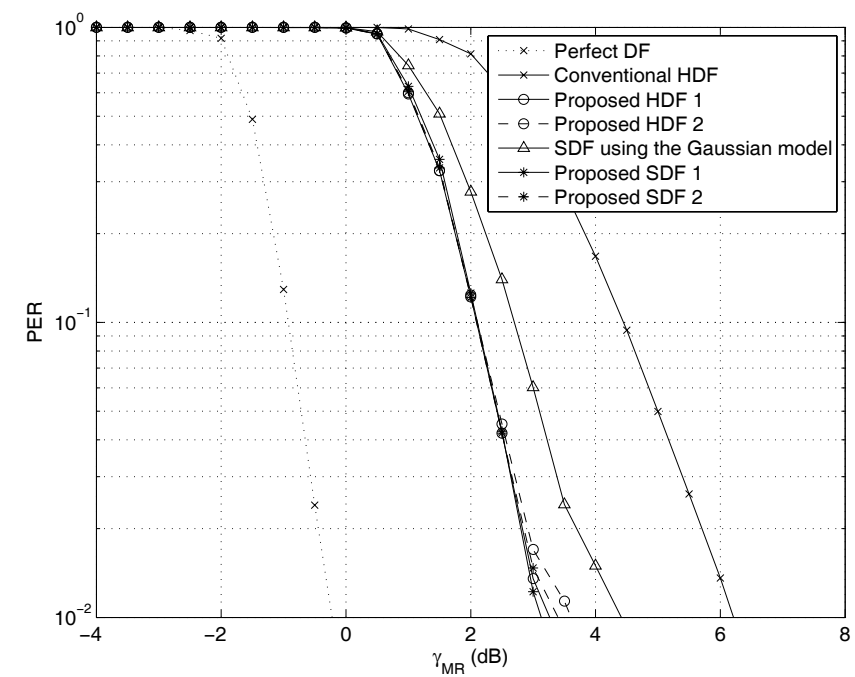

Fig. 6. PER versus SNR performance over a frequency-flat channel using $N_{t}=2, N_{r}=2, \gamma_{\text {diff }}=0 \mathrm{~dB}$.

detect the hard-valued error $\Delta x_{A, k}^{h}$. Furthermore, in the high SNR region, the hard-valued errors rarely occur and we do not have enough samples to estimate $\sigma_{e, 2}^{2}$. As mentioned in Section III-B, instead of separately estimating $\sigma_{e, 1}^{2}$ and $\sigma_{e, 2}^{2}$, the combined parameter $\sigma_{e}^{2}$ can be used, which results in the modified distribution model

$$
\begin{gathered}
f\left(\Delta x_{A, k}\right)=(1-q) \cdot g\left(\Delta x_{A, k} \mid 0, \sigma_{e}^{2}\right) \\
+q \cdot g\left(\Delta x_{A, k} \mid \sqrt{2}, \sigma_{e}^{2}\right)+q \cdot g\left(\Delta x_{A, k} \mid-\sqrt{2}, \sigma_{e}^{2}\right), \\
-\sqrt{2} \leq \Delta x_{A, k} \leq \sqrt{2} .
\end{gathered}
$$

In Fig. 5, the probability distribution of (35), which is termed as the proposed distribution model 2 , is also plotted. The parameter $\sigma_{e}^{2}$ is estimated by the algorithm outlined in Section III-C.

We observe that the proposed error models are more accurate than the Gaussian distribution, especially for error values above 0.7 and these values are more influential in determining the true error probability. Hence we anticipate that this model may provide a performance improvement compared to the Gaussian error model, which was employed in [14]. It is also seen that the proposed distribution model 2 is similar to the proposed distribution model 1 . In the following simulation results, the proposed distribution model 2 is employed, since it is practically more realizable. Furthermore, by adopting the proposed distribution model 2, the computational complexity can also be reduced, as mentioned in Section III-B.

Figs. 6-9 characterize the packet error ratio (PER) performance of various decoders, which is defined synonymously to the codeword error rate. The perfect $D F$ denotes the DF relaying scheme that transmits the perfectly decoded packet from the RS. More specifically, it does not suffer from decoding errors at the RS and hence it naturally outperforms the realistic relaying schemes. By contrast, the conventional $H D F$ represents the specific HDF scheme, which does not consider the potential presence of decoding errors at the RS. More explicitly, the decoder at the BS or the MS of conventional HDF relaying always assumes the presence of perfectly decoded data, which are transmitted from the RS,

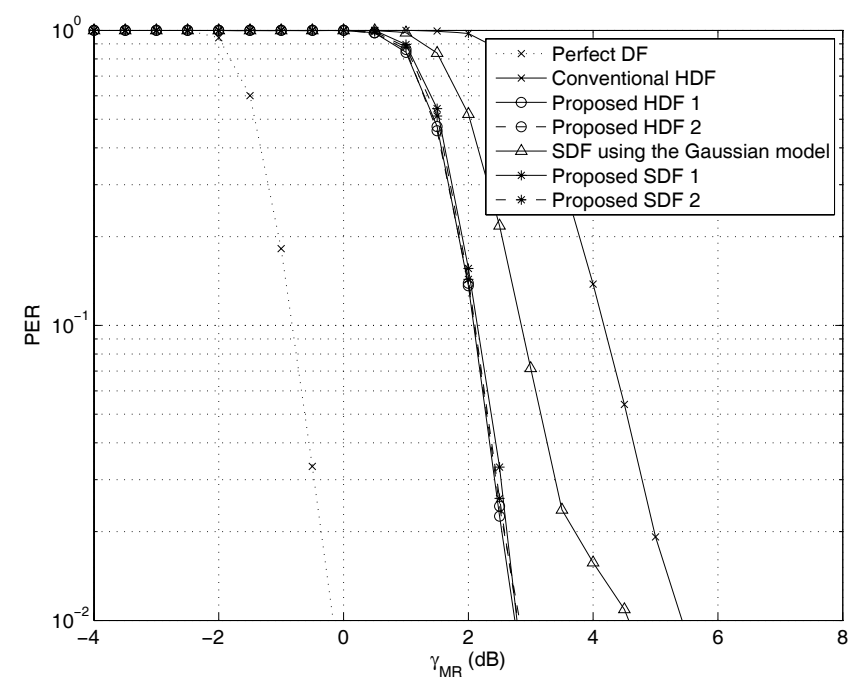

Fig. 7. PER versus SNR performance over a frequency-flat channel using $N_{t}=4, N_{r}=4, \gamma_{\text {diff }}=0 \mathrm{~dB}$.

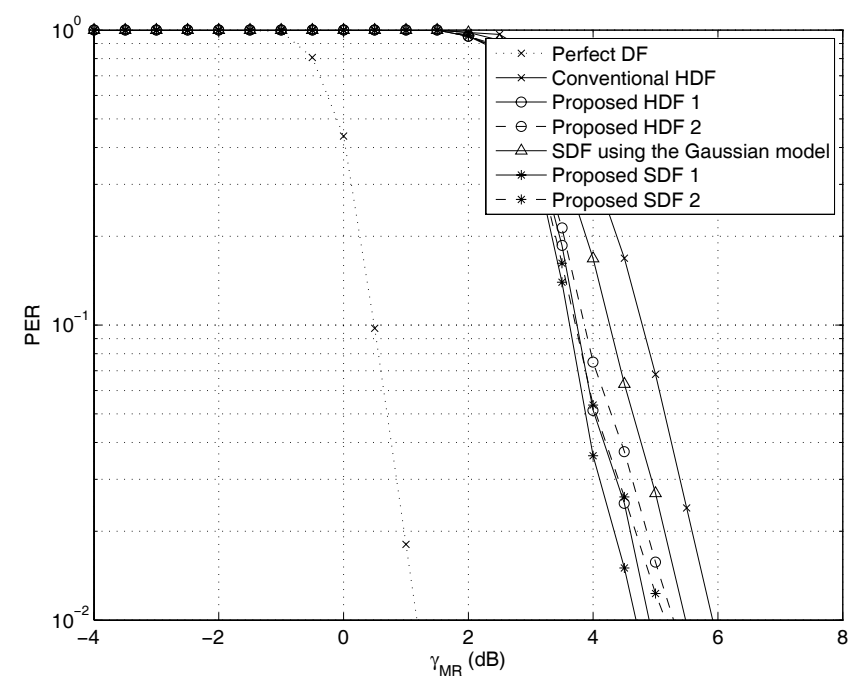

Fig. 8. PER versus SNR performance over a frequency-flat channel using $N_{t}=4, N_{r}=4, \gamma_{d i f f}=3 \mathrm{~dB}$.

regardless whether the forwarded packet actually contains errors. The idealized HDF and SDF decoders assuming the perfect knowledge of $q$ and $\sigma_{e}$ are referred to as Proposed HDF 1 and Proposed SDF 1 schemes, respectively. The proposed decoders that generate realistic estimates of $q$ and $\sigma_{e}$ as described in Section III-C are referred to as Proposed HDF 2 and Proposed SDF 2. The SDF using the Gaussian model represents the relaying system, where the expectation values of the symbols are transmitted from the RS to both the MS and BS to minimize the mean square error of the forwarded signal. Then again, the Gaussian error model is assumed for supporting the decoder's operation at the destination nodes, as proposed in [14].

Fig. 6 illustrates the PER performance of NCR systems using $N_{t}=2, N_{r}=2$, and $\gamma_{\text {diff }}=0 \mathrm{~dB}$. It is observed that the proposed decoders using the error distribution of (35) have a better performance compared to the decoder assuming the Gaussian error model. Interestingly, the proposed HDF 


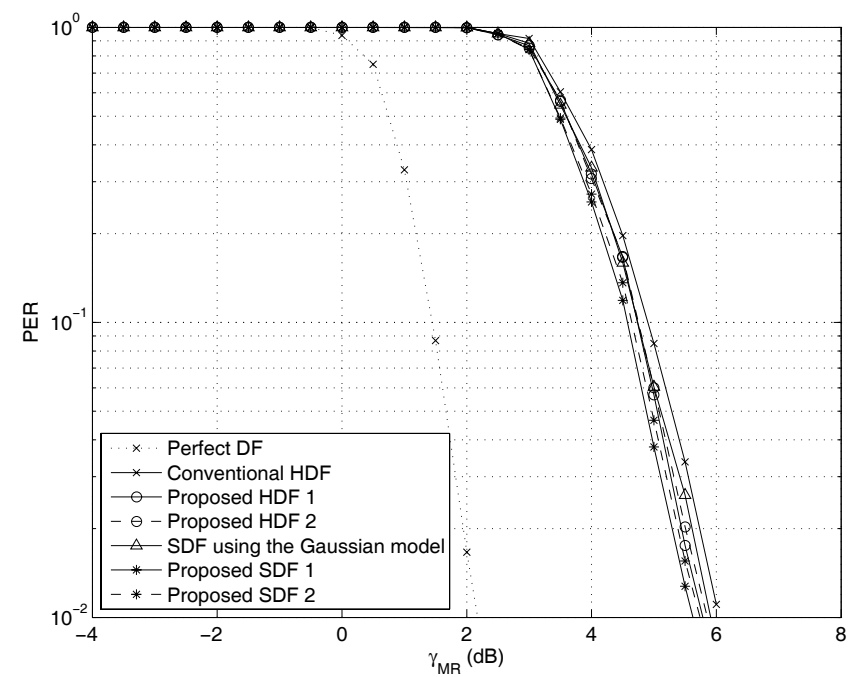

Fig. 9. PER versus SNR performance over a frequency-flat channel using $N_{t}=4, N_{r}=4, \gamma_{\text {diff }}=6 \mathrm{~dB}$.

decoders also have a better performance than the SDF decoder of [14] despite its lower complexity. This is because the proposed HDF decoder employs an accurate hard error model. The SDF reduces the mean square error of the symbol to be forwarded, but the relatively inaccurate error model results in the observed performance degradation. It is also seen in Fig. 6 that the proposed SDF decoders have a slightly better performance than the proposed HDF decoders. In the lowSNR region, the decoders using the estimated parameters exhibit a similar performance to those associated with the perfect knowledge of the parameters $q$ and $\sigma_{e}$. In the highSNR region, they perform slightly worse, but their SNR disadvantage is less than $0.4 \mathrm{~dB}$.

In Fig. 7, the performance of the $N_{t}=4, N_{r}=4$, and $\gamma_{\text {diff }}=0 \mathrm{~dB}$ scenario is characterised. Fig. 7 demonstrates that the proposed decoders provide a substantial performance improvement compared to both the conventional HDF decoder and to the SDF decoder using the Gaussian error model. At $\mathrm{PER}=10^{-2}$, the proposed decoders achieve an approximately $1.8 \mathrm{~dB}$ SNR gain over the SDF decoder assuming the Gaussian error model.

Figs. 8-9 illustrate the achievable PER performance, when the relayed link has a higher SNR than the direct link. Fig. 8 characterises the PER versus SNR performance of NCR systems associated with $N_{t}=4, N_{r}=4$, and $\gamma_{\text {diff }}=3$ $\mathrm{dB}$, where the proposed decoders outperform the conventional HDF decoder and the SDF decoder using the Gaussian model although the SNR gains of the proposed decoders decrease, as $\gamma_{d i f f}$ increases. We also arrive at the same conclusion from Fig. 9, which characterises the performance of $N_{t}=4, N_{r}=$ 4 , and $\gamma_{\text {diff }}=6 \mathrm{~dB}$. It is observed in Fig. 9 that the SNR gains of the proposed decoders become lower for higher $\gamma_{\text {diff } f}$, but their performances are still superior to that of the decoder of [14].

In Figs. 7-9, it is also seen that the proposed SDF 1 scheme's gain over the other proposed decoders is maximized at approximately $\gamma_{d i f f}=3 \mathrm{~dB}$. For $\gamma_{d i f f}=0 \mathrm{~dB}$, the directly received signal, which has the same SNR as the relayed signal, gravely affects the achievable decoding performance. Furthermore, the performance difference between the proposed decoders, which is caused by the modeling inaccuracy as well as the imperfect knowledge of parameters becomes relatively small. By contrast, for $\gamma_{d i f f}=3 \mathrm{~dB}$, the direct link has a lower SNR and hence the accuracy of the relayed signal and related parameters, which depends on the specific SDF and HDF scheme considered, starts to substantially affect the attainable end-to-end performance. Finally, for $\gamma_{d i f f}=6 \mathrm{~dB}$, the direct link has a significantly lower SNR than the relay link and hence the decoding errors at the relay cannot be efficiently mitigated with the aid of the side information obtained via the direct link. Therefore, in this case, even if the decoding errors of the RS are carefully considered at the receiver, the overall performance converges to that of the worst case scenario, where the effects of the decoding errors are not considered at all. Consequently, all the relaying schemes perform similarly for $\gamma_{d i f f}=6 \mathrm{~dB}$ and the maximum gain of the proposed $S D F$ 1 scheme is observed at the medium values of $\gamma_{\text {diff }}$.

\section{CONCLUSION}

In this paper, we have derived error models for NCR systems using multiple antennas. First, we considered the HDF relaying scheme and proposed a new decoding algorithm, which takes the estimated bit error rate of the forwarded packet into account, when performing iterative detection and decoding at both the BS and the MS. More explicitly, we obtained a simplified expression for the extrinsic LLR at the output of the MIMO detector. Secondly, a new decoder has been derived for SDF relaying. In (26), we modeled the error of the forwarded signal as the combination of the hard and soft errors, and developed the HDF scheme's decoder for employment in SDF in conjunction with a modified cost function. Finally, we proposed realistic estimation algorithms to acquire the parameters necessary for the operation of the proposed decoders.

The simulation results of Figs. 6-9 show that both the proposed HDF and SDF decoders achieve a better performance compared to the decoders previously proposed in the open literature. In the NCR system using $N_{t}=4, N_{r}=4$, and $\gamma_{\text {diff }}=0$, the proposed decoders provide around $1.8 \mathrm{~dB}$ SNR gain at $P E R=10^{-2}$ with respect to their benchmarker using the Gaussian error model.

The proposed error model of the SDF in (26) is constituted by the combination of the hard and Gaussian soft errors and hence it is more accurate than the simple Gaussian error model. However, the proposed SDF decoder shows only small SNR gains compared to the HDF decoder because the error model is still insufficiently accurate, as seen in Fig. 5. Therefore, the further study of a new error model may be a possible way of improving the attainable decoding performance of relaying systems.

\section{REFERENCES}

[1] L. Hanzo, C. H. Wong, and M. S. Yee, Adaptive Wireless Transceivers. New York: Wiley, IEEE Press, 2002.

[2] A. Sendonaris, E. Erkip, and B. Aazhang, "User cooperation diversitypart I: system description," IEEE Trans. Commun., vol. 51, pp. $1927-$ 1938, Nov. 2003. 
[3] A. Sendonaris, E. Erkip, and B. Aazhang, "User cooperation diversitypart II: implementation aspects and performance analysis," IEEE Trans. Commun., vol. 51, pp. 1939-1948, Nov. 2003.

[4] J. N. Laneman, D. N. C. Tse, and G. W. Wornell, "Cooperative diversity in wireless networks: efficient protocols and outage behavior," IEEE Trans. Inform. Theory, vol. 50, pp. 3062-3080, Dec. 2004.

[5] R. Pabst, B. H. Walke, D. C. Schultz, P. Herhold, H. Yanikomeroglu, S. Mukherjee, H. Viswanathan, M. Lott, W. Zirwas, M. Dohler, H. Aghvami, D. D. Falconer, and G. P. Fettweis, "Relay-based deployment concepts for wireless and mobile broadband radio," IEEE Commun. Mag., vol. 42, pp. 80-89, Sept. 2004

[6] P. Liu, Z. Tao, Z. Lin, and E. Erkip, "Cooperative wireless communications: a cross-layer approach," IEEE Wireless Commun., vol. 13, pp. 84-92, Aug. 2006.

[7] P. Larsson, N. Johansson, and K.-E. Sunell, "Coded bi-directional relaying," in Proc. IEEE Veh. Technol. Conf., Melbourne, Australia, May 2006, pp. 851-855.

[8] L. Xiao, T. Fuja, J. Kliewer, and D. J. Costello, "A network coding approach to cooperative diversity," IEEE Trans. Inform. Theory, vol. 53, pp. 3714-3722, Oct. 2007.

[9] B. Rankov and A. Wittneben, "Achievable rate regions for the two-way relay channel," in Proc. IEEE Inter. Symp. Inform. Theory (ISIT-2006), Seattle, USA, July 2006, pp. 1668-1672.

[10] T. J. Oechtering and H. Boche, "Optimal transmit strategies in multiantenna bidirectional relaying," in Proc. IEEE Inter. Conf. Acoustics, Speech Signal Processing (ICASSP-2007), Hawaii, USA, Apr. 2007, vol. 3, pp. $145-148$.

[11] I. Hammerström, M. Kunhn, C. Esli, J. Zhao, A. Wittneben, and G. Bauch, "MIMO two-way relaying with transmit CSI at the relay," in Proc. IEEE 8th Workshop Signal Processing Advances Wireless Commun. (SPAWC-2007), Helsinki, Finland, June 2007, pp. 17-20.

[12] G. Kramer, M. Gastpar, and P. Gupta, "Cooperative strategies and capacity theorems for relay networks," IEEE Trans. Inform. Theory, vol. 51, pp. 3037-3063, Sept. 2005.

[13] H. H. Sneessens and L. Vandendorpe, "Soft decode and forward improves cooperative communications," in Proc. IEEE International Workshop Computational Advances in Multi-Sensor Adaptive Processing, Puerto Vallarta, Mexico, Dec. 2005, pp. 157-160.

[14] Y. Li, B. Vucetic, T. F. Wong, and M. Dohler "Distributed turbo coding with soft information relaying in multihop relay networks," IEEE $J$. Select. Areas Commun., vol. 24, pp. 2040-2050, Nov. 2006.
[15] B. M. Hochwald and S. ten Brink, "Achieving near-capacity on a multiple-antenna channel," IEEE Trans. Inform. Theory, vol. 51, pp. 389399, Mar. 2003.

[16] H. Vikalo, B. Hassibi, and T. Kailath, "Iterative decoding for MIMO channels via modified sphere decoding," IEEE Trans. Wireless Commun., vol. 3, pp. 2299-2311, Nov. 2004.

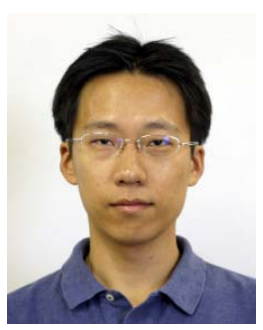

Kyungchun Lee received the B.S., M.S. and Ph.D. degrees in electrical engineering from Korea Advanced Institute of Science and Technology (KAIST), Daejeon, in 2000, 2002, and 2007, respectively. From September 2005 until February 2006, he was a visiting research student with the Communication Research Group, University of Southampton, U.K, where he held a post-doctorial visiting research fellowship from April 2007 until June 2008. Currently, he is with Samsung Electronics, Suwon, Korea. His research interests include signal processing for wireless communications with focus on MIMO and relay systems.

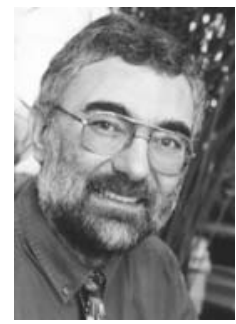

Lajos Hanzo FREng, FIEEE, FIET, DSc received his degree in electronics in 1976 and his doctorate in 1983. During his career in telecommunications he has held various research and academic posts in Hungary, Germany and the UK. Since 1986 he has been with the School of Electronics and Computer Science, University of Southampton, UK, where he holds the chair in telecommunications. He has coauthored 17 books on mobile radio communications totalling in excess of 10000 , published about 800 research papers, acted as TPC Chair of IEEE conferences, presented keynote lectures and been awarded a number of distinctions. Currently he is directing an academic research team, working on a range of research projects in the field of wireless multimedia communications sponsored by industry, the Engineering and Physical Sciences Research Council (EPSRC) UK, the European Community and the Mobile Virtual Centre of Excellence (VCE), UK. He is an enthusiastic supporter of industrial and academic liaison and he offers a range of industrial courses. He is also an IEEE Distinguished Lecturer as well as a Governor of both ComSoc and the VTS. He is acting as Editor-in-Chief of the IEEE Press. For further information on research in progress and associated publications please refer to http://www-mobile.ecs.soton.ac.uk 